\title{
Splenic Rupture mimicking Dengue Shock Syndrome
}

\section{Introduction}

Although 50-100 million people are infected by dengue annually, ${ }^{1}$ and $9-60 \%$ of the cases develop dengue shock syndrome (DSS), ${ }^{2}$ only a mere 9 cases of spontaneous splenic rupture have been reported in literature. ${ }^{1,3,5-10} \mathrm{~A}$ misdiagnosis of splenic rupture for DSS can lead to inappropriate management of the patient with high risk of attendant mortality. We report a case of ruptured spleen in dengue that was initially suspected to be DSS.

\section{Case report}

A 22-year-old male presented to a community hospital with a 5 day history of fever and headache. He was assessed and found stable and was sent home on conservative management. The following day he returned to the hospital with complaints of high fever, worsening abdominal pain, bilious vomiting and diarrhea. He had also begun to experience dyspnea. He was admitted to the hospital and serology for dengue was found to be positive. The platelet count was $78 \times 10^{9} / \mathrm{L}$. The dyspnea worsened overnight, with a respiratory rate of 28 breaths per minute. In addition, the patient's blood pressure fell to 90/50 $\mathrm{mmHg}$ with a pulse rate of $120 / \mathrm{min}$. Dengue shock syndrome was suspected. However 12 hours later the patient's abdominal pain worsened and he complained of increasing abdominal distension. Examination revealed a tense, tender, distended abdomen. Computed tomography scan of the abdomen revealed a ruptured spleen with hemoperitoneum (Figure 1). The patient's platelet count at this juncture had fallen to $53 \times 10^{9} / \mathrm{L}$ and his hemoglobin had dropped to $5 \mathrm{~g} / \mathrm{dl}$, from baseline $16 \mathrm{~g} /$ $\mathrm{dl}$ recorded at admission. An emergency exploratory laparotomy was performed which revealed a hemoperitoneum with 3 litres of blood and clots. The ruptured spleen was removed (Figure 2) and the raw surface packed with 6 laparotomy pads because of persistent diffuse oozing. The next day patient's platelet count increased to $136 \times 10^{9} / \mathrm{L}$ and on the second postoperative day it was $265 \times 10^{9} / \mathrm{L}$. At this time he was re-explored and the packs removed, with no remnant signs of active bleeding. The patient's recovery was uncomplicated and he was discharged 5 days postoperatively.

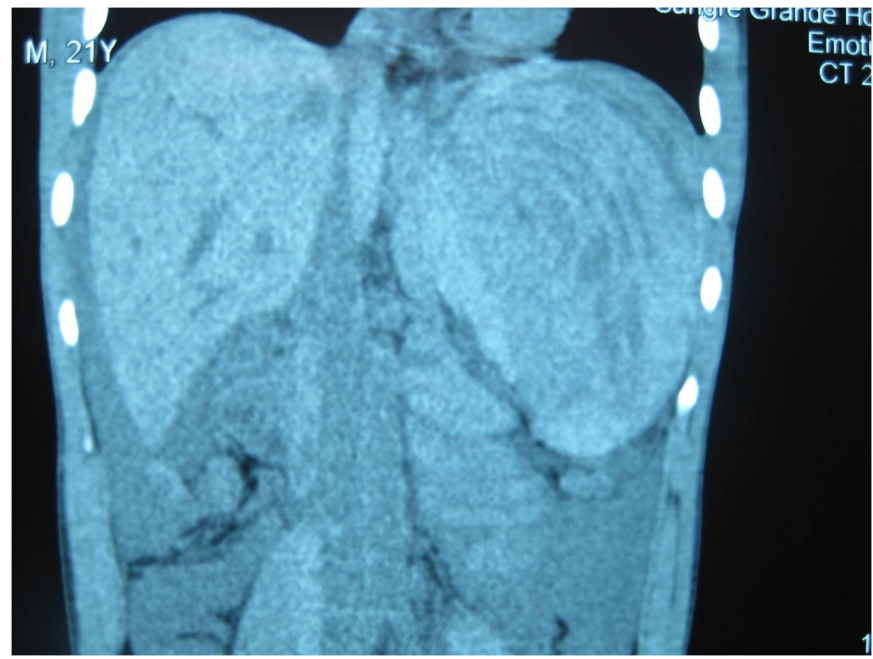

Figure 1: Computed Tomography scan of ruptured spleen and hemoperitoneum

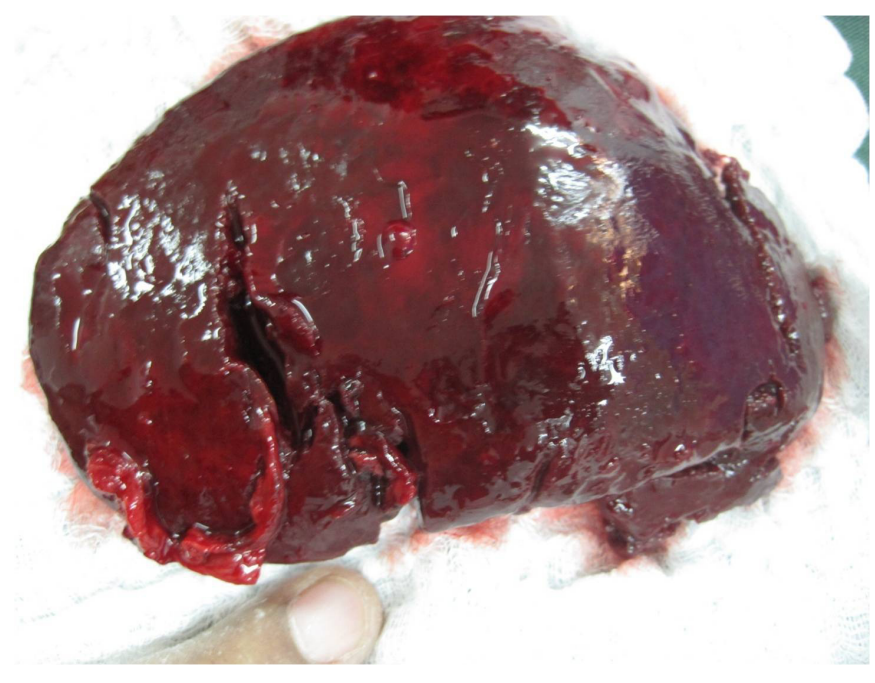

Figure 2: Ruptured spleen after removal

\section{Discussion}

The underlying mechanism of splenic rupture in infectious diseases is subcapsular hemorrhage due to vascular abnormalities and thrombocytopenia. ${ }^{3}$ The spleen, which is frequently congested, bears subcapsular hematomas in $15 \%$ of dengue cases. ${ }^{4}$ The hypotension and tachycardia of splenic rupture can be easily misdiagnosed as the more common DSS. ${ }^{5}$ However, in DSS the hemoglobin and hematocrit tend to rise while in splenic rupture they decrease, as seen in our case. ${ }^{11,12}$ Therefore spontaneous splenic rupture can be a fatal complication in cases of dengue fever, if not quickly and correctly diagnosed. ${ }^{6}$ Accordingly physicians should be aware of the possibility of splenic rupture in areas where dengue fever is endemic, because a timely splenectomy can be curative and life saving as was in our case. Moreover, even in the 
presence of thrombocytopenia splenectomy can be performed with packing of the raw surface as a temporary hemostatic measure. Gauze packing has been used successfully to achieve hemostasis during splenic rupture in the presence of coagulopathy. ${ }^{13}$ Its noteworthy that the removal of the ruptured spleen not only stopped the bleeding but also afforded a remarkable rise in the platelet count postoperatively, thereby facilitating safe removal of the hemostatic packs 2 days later.

$\mathrm{P}_{\text {GOPIE }}{ }^{1}$

$S$ TEELUCKSINGH ${ }^{2}$ V NARAYNSINGH ${ }^{1}$

Correspondence: Dr. Priya Gopie Departments of Clinical Surgical Sciences ${ }^{1}$ and Clinical Medical Sciences, ${ }^{2}$ University of the West Indies, Champ Fleurs, Trinidad \& Tobago Email:priyagopie@hotmail.com

\section{References}

1. Seravali MR, Santos AH, Costa CE, Rangel DT, Valentim LF, Gonçalves RM. Spontaneous splenic rupture due to dengue fever: report of two cases. Braz J Infect Dis. 2008;12:538-40.

2. World Health Organization. Available at http://whqlibdoc.who.int/ hq/2005/WHO_FCH_CAH_05.13_eng.pdf

3. Imbert P, Sordet D, Hovette P, Touze JE. Spleen rupture in a patient with dengue fever. Trop Med Parasitol. 1993;44:327-8.

4. Bhamarapravati N, Tuchinda P, Boonyapaknavik V. Pathology of Thailand haemorrhagic fever: a study of 100 autopsy cases. Ann Trop Med Parasitol. 1967;61:500-10.

5. Miranda LE, Miranda SJ, Rolland M. Case report: spontaneous rupture of the spleen due to dengue fever. Braz J Infect Dis. 2003;7:423-5.

6. Pungjitprapai A, Tantawichien T. A fatal case of spontaneous rupture of the spleen due to dengue virus infection: case report and review. Southeast Asian J Trop Med Public Health. 2008;39:383-6.

7. Sharma SK, Kadhiravan T. Spontaneous splenic rupture in dengue hemorrhagic fever. Am J Trop Med Hyg. 2008;78:7.

8. Rapp C, Debord T, Imbert P, Lambotte O, Roué R. Splenic rupture in infectious disease: splenectomy or conservative treatment? Report of three cases. Rev Med Interne. 2002;23:85-91.

9.. Couvelard A, Marianneau P, Bedel C, Drouet MT, Vachon F, Hénin D, Deubel V. Report of a fatal case of dengue infection with hepatitis: demonstration of dengue antigens in hepatocytes and liver apoptosis. Hum Pathol. 1999;30:1106-10.

10. Redondo MC, Ríos A, Cohen R, Ayala J, Martínez J, Arellano G, Paz V, et al. Hemorrhagic dengue with spontaneous splenic rupture: case report and review. Clin Infect Dis. 1997;25:1262-3.

11. Ali N, Usman M, Syed N, Khurshid M. Haemorrhagic manifestations and utility of haematological parameters in dengue fever: a tertiary care centre experience at Karachi. Scand J Infect Dis. 2007;39:1025-8.
12. Dengue haemorrhagic fever: diagnosis, treatment, prevention and control. Clinical diagnosis. 2nd ed. Geneva : World Health Organization; 1997. p.12-23.

13. Naraynsingh V, Maharaj D, Ramdass MJ. Gauze packing and planned reoperation for splenic trauma in the presence of coagulopathy. Internet J Surg. 2001; 2.

\section{A rare case of gallbladder carcinoma metastases to the breast treated with curative intent}

\section{Introduction}

Carcinoma of the gallbladder is one of the most common malignancies in north India, particularly in females. ${ }^{1}$ Majority of them are metastatic or unresectable at the time of presentation. The modes of dissemination in metastatic gallbladder carcinoma are lymphatic, vascular, neural, intraperitoneal and intraductal. Liver and lymph nodes are the two most common sites of dissemination. ${ }^{2}$ To our knowledge, solitary breast metastasis is an unusual site of dissemination and till date only two cases have been reported in the literature. Given the unusual nature of these metastases of gallbladder cancer, diverse management strategies have been employed without any proper consensus guidelines.

\section{Case report}

A 35-year-old female had undergone laparoscopic cholecystectomy for gallstones in January 2007 in a private hospital. The histopathology showed an incidental gallbladder adenocarcinoma stage T2NOM0. The patient defaulted further treatment for the cancer. Subsequently she noticed an abdominal wall subcostal port site lump in June 2008. The incisional biopsy showed metastatic adenocarcinoma. The patient was referred to our institution at this juncture, for further management. On evaluation contrast enhanced tomography showed a $3 \times 3 \mathrm{~cm}$ mass at the epigastric port site with no evidence of metastasis elsewhere. The patient underwent explorative laparotomy with wide excision of the port site recurrence. The intra-operative findings showed neither recurrence in the gallbladder fossa nor any regional lymphadenopathy or associated metastasis. Histopathology of the specimen showed an infiltrating adenocarcinoma with 